\title{
Yukawa particles confined in a channel and subject to a periodic potential: Ground state and normal modes
}

\author{
J. C. N. Carvalho,,$^{1, *}$ W. P. Ferreira, ${ }^{1, \dagger}$ G. A. Farias, ${ }^{1, \ddagger}$ and F. M. Peeters ${ }^{1,2, \S}$ \\ ${ }^{1}$ Departamento de Física, Universidade Federal do Ceará, Caixa Postal 6030, Campus do Pici, 60455-760 Fortaleza, Ceará, Brazil \\ ${ }^{2}$ Department of Physics, University of Antwerp, Groenenborgerlaan 171, B-2020 Antwerpen, Belgium
}

(Received 9 June 2010; revised manuscript received 6 January 2011; published 8 March 2011)

\begin{abstract}
We consider a classical system of two-dimensional (2D) charged particles, interacting through a repulsive Yukawa potential $\exp (-r / \lambda) / r$, and confined in a parabolic channel that limits the motion of the particles in the $y$ direction. Along the $x$ direction, the particles are subject to a periodic potential. The ground-state configurations and the normal-mode spectra of the system are obtained as a function of the periodicity and strength of the periodic potential $\left(V_{0}\right)$ and density. An interesting set of tunable ground-state configurations are found, with firstor second-order structural transitions between them. A configuration with particles aligned, perpendicular to the $x$ direction, in each minimum of the periodic potential is obtained for $V_{0}$ larger than some critical value that has a power-law dependence on the density. The phonon spectrum of different configurations was also calculated. A localization of the modes into a small frequency interval is observed for sufficiently large strength of the periodic potential, and a tunable gap in the phonon spectrum is found as a function of $V_{0}$.
\end{abstract}

DOI: 10.1103/PhysRevB.83.094109

PACS number(s): 64.60.Cn, 82.70.Dd, 63.20.D-

\section{INTRODUCTION}

A two-dimensional system (2D) is often created in the presence of a substrate, ${ }^{1}$ which may induce a periodic potential on the particles. In the pioneering experimental work of Chowdhury et al., ${ }^{2}$ a 2D colloidal system under the influence of a one-dimensional (1D) periodic potential was studied. An optical tweezer has been used to trap the colloids by laser beams. For very high values of the light intensity, crystallization of the colloidal suspension was observed in the case in which the periodicity of the substrate (i.e., the periodic potential) was commensurate with the mean particle distance. Laser-induced freezing, which is caused by the suppression of thermal fluctuations transverse to the 1D periodic substrate, was found (liquid-solid transition). ${ }^{3}$ The system studied in Ref. 2 is related to a colloidal molecular crystal (CMC) which received attention recently due to important applications in photonic and phononic crystals. $^{4-6}$

Specifically, CMC occurs when the number of colloids is an integer multiple of the number of substrate minima, which has been investigated numerically ${ }^{5,7}$ and realized experimentally. ${ }^{8}$ CMC is an interesting experimental system to study order and the dynamics in 2D since the typical particle size and relaxation times permit the use of digital video-microscopy to track particle trajectories, allowing a deeper study of the physical behavior of the system. ${ }^{9}$

Originally, CMC was proposed for a $2 \mathrm{D}$ system in the presence of a 2D periodic potential (substrate). As is known, the dimensionality of the system plays an important role in many physical properties of distinct physical phenomena. In this sense, an interesting question is how the ordered structures and physical properties would be influenced by the dimensionality of the periodic substrate. Recently, HerreraVelarde and Priego ${ }^{10,11}$ studied a 1D system of repulsive colloidal particles subject to an external 1D periodic potential, which is thus the $1 \mathrm{D}$ version of the $\mathrm{CMC}$. The main focus of this study was the role of the substrate on the mechanisms that lead to a variety of commensurate and noncommensurate phases, its effect on the the single-file diffusion regime, and the pinning-depinning transition.

In the present paper, we study the ordered configurations and the phonon spectrum of a 2D system of repulsive (Yukawa interaction) particles confined in a parabolic channel and subjected to a $1 \mathrm{D}$ periodic potential along the channel. Our study is a generalization of Refs. 10 and 11 to quasi-1D (Q1D) in the sense that particles are still allowed to move freely in the perpendicular direction of the confinement potential. This extra degree of freedom leads to a much higher set of possible ground-state configurations.

The interplay between the repulsive interparticle interaction and the periodic potential results in different ground-state configurations. For example, in the present paper we found a structural phase transition where the number of particles in the unit cell is changed. This implies that the number of phonon branches changes, and this is an interesting feature relevant for applications in phononics. In general, the configurations can be tuned according to the strength of the periodic substrate $V_{0}$, leading to arrangements with controlled porosity. We find in some cases that for a critical $V_{0}$, particles become aligned along the $y$ direction in each minimum of the substrate. For such a configuration, we find that the normal-mode frequencies are independent of the wave vector, and thus the normal mode does not propagate along the channel.

Our model system of Yukawa particles can be realized experimentally using (i) a dusty plasma, ${ }^{12-14}$ (ii) colloidal systems, ${ }^{15,16}$ and (iii) electrons on liquid helium. ${ }^{17,18}$ A dusty plasma consists of interacting microscopic dust particles immersed in an electron-ion plasma. The dust particles acquire a net charge and the Coulomb interaction between the dust particles is shielded by the electron-ion plasma resulting in a Yukawa or screened Coulomb interparticle interaction. The dust particles are confined to a two-dimensional layer through a combination of gravitational and electrical forces. By microstructuring a channel in the bottom electrode of the discharge, it is possible to laterally confine the dust particles, as was realized in Refs. 19-23. The strength of the 1D 
confinement potential can be varied by the width of the channel or the potential on the bottom electrode. When the width of the channel is microstructured into an oscillating function along the channel, it will result in a periodic potential along the channel.

Alternatively, one can confine charged colloids, which move in a liquid environment containing counterions, into microchannels, as was recently realized experimentally in Ref. 24. In this case, the intercolloid interaction can be modeled by a screened Coulomb interaction and the confinement potential is a hard-wall potential. By changing the depth profile of the microchannel, it was shown in Ref. 25 that the confinement potential can be tuned into a harmonic potential. Microstructuring the width of the channel into an oscillating function along the channel will result in an additional periodic potential along the channel.

In a previous work, ${ }^{26}$ the ordered configurations of Yukawa particles confined to Q1D were studied. A phase diagram was obtained as a function of the particle density and the inverse Debye screening length, which is a measure of the strength of the interparticle interaction. The competition between the lateral confinement and the screened Coulomb interaction resulted in different phases where the particles are ordered in chains. The most well studied phases are the one- and two-chain configurations, where the transition between those two phases occurs through a zigzag transition. The latter is a continuous transition as found theoretically for mono- ${ }^{27}$ and bidisperse ${ }^{28,29}$ systems, and experimentally ${ }^{22,23}$ with a powerlaw dependence on the width. ${ }^{27,30}$ Here we are interested to investigate how the phase diagram will be modified when an additional 1D periodic potential is present. For example, how will the zigzag transition be modified by the periodic potential?

The present paper is organized as follows. In Sec. II, we describe the model system and methods used in the calculation of the properties. In Sec. III, we present the results for the different ground-state configurations. In Sec. IV, the normal-mode spectra for the one and two-chain regimes are presented for different intensities of the periodic potential. Our conclusions are given in Sec. V.

\section{THE MODEL}

Our system consists of identical pointlike particles interacting through a screened Coulomb potential. The particles are allowed to move in a $2 \mathrm{D}$ plane and are subject to an external parabolic confinement in the $y$ direction and a periodic substrate potential along the $x$ direction. A sketch of the present model system is shown in Fig. 1. The total interaction energy of the system is given by

$\mathrm{H}^{\prime}=\frac{q^{2}}{\varepsilon} \sum_{i<j} \frac{e^{-\left|\overrightarrow{r_{i}^{\prime}}-\overrightarrow{r_{j}^{\prime}}\right| / \lambda}}{\left|\overrightarrow{r_{i}^{\prime}}-\overrightarrow{r_{j}^{\prime}}\right|}+\sum_{i} \frac{1}{2} m \omega_{0}^{2} y_{i}^{\prime 2}+V_{0}^{\prime} \sum_{i} \cos \left(\frac{2 \pi x_{i}^{\prime}}{L}\right)$,

where $\epsilon$ is the dielectric constant of the medium in which the particles are moving, $\lambda$ is the Debye screening length, $V_{0}^{\prime}$ is the strength of the periodic substrate potential, $L$ is the periodicity of the substrate potential, and $\mathbf{r}_{i}^{\prime}=\left(x_{i}^{\prime}, y_{i}^{\prime}\right)$ is the position of the $i$ th particle. To keep in Eq. (1) only the parameters that rule the physics of the system, it is convenient to write the energy

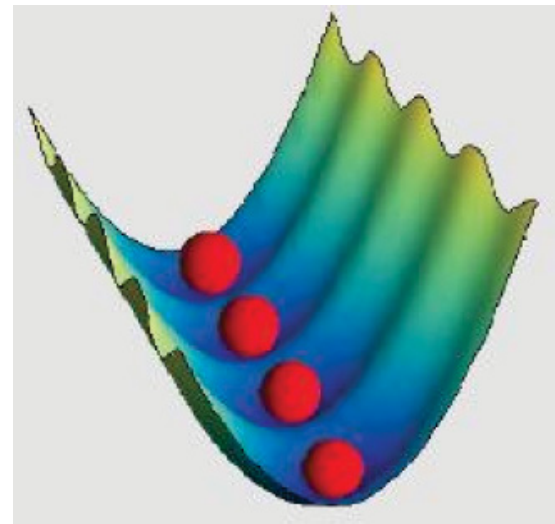

FIG. 1. (Color online) A sketch of the model system.

and the distances in units of $E_{0}=\left(m \omega_{0}^{2} q^{4} / 2 \epsilon^{2}\right)^{1 / 3}$ and $r_{0}=$ $\left(2 q^{2} / m \epsilon \omega_{0}^{2}\right)^{1 / 3}$, respectively, and the screening parameter as $\kappa=r_{0} / \lambda$. We also define the dimensionless strength of the substrate potential $V_{0}=V_{0}^{\prime} / E_{0}$ and $\vec{r}=\vec{r}^{\prime} / r_{0}$. In so doing, the expression for the energy is reduced to

$$
H=\sum_{i<j} \frac{e^{-\kappa\left|\vec{r}_{i}-\vec{r}_{j}\right|}}{\left|\vec{r}_{i}-\vec{r}_{j}\right|}+\sum_{i} y_{i}^{2}+V_{0} \sum_{i} \cos \left(\frac{2 \pi x_{i}}{L}\right) .
$$

As can be observed from Eq. (2), the system is a function of the parameters $\kappa, V_{0}, L$, and the density. In our numerical calculations, $\kappa=1$, which is a typical value for dusty plasma and colloidal systems. We introduce the distance $a_{0}$, which is defined as the distance between particles along the 1D chain when $V_{0}=0$. The density $(n)$ is the ratio between the number of chain $N_{\text {ch }}$ and $a_{0}$, that is, $n=N_{\mathrm{ch}} / a_{0}$. In the case $V_{0}=0$, the system self-organizes in a multichain-like structure. ${ }^{26}$

The model studied in this work is related to the FrenkelKontorova (FK) model, which is a simple 1D model that describes the dynamics of a chain of particles interacting with nearest neighbors in the presence of an external periodic substrate potential. This model was initially introduced in the 1930 s by Frenkel and Kontorova ${ }^{31}$ and was subsequently reinvented independently by others, notably Frank and Van der Merwe. ${ }^{32}$ It provides a simple and realistic description of commensurate-incommensurate transitions when thermal fluctuations are insignificant, therefore the system energy is only characterized by the potential energy. The expression for the potential energy of this one-dimensional model is given by

$$
U=\sum_{i}\left[\frac{1}{2} K\left(x_{i+1}-x_{i}-a\right)^{2}+V\left(x_{i}\right)\right],
$$

where $x_{i}$ and $a$ are the position of the $i$ th particle and the natural equilibrium distance between the particles, respectively. The first (elastic) term in Eq. (3) takes into account a linear coupling between the nearest neighbors, while the second, $V\left(x_{i}\right)$, is an arbitrary function with period $L$, usually described as $V(x)=$ $-V_{0} \cos (2 \pi x / L)$. The main feature of this model consists in the competition between the interparticle interaction and the substrate periodic potential.

The FK model and our model system are similar, so some structural properties already found in the FK model ${ }^{32}$ are expected to be observed. However, differently from the FK model, we consider particles interacting not only with 
nearest neighbors, but also with further neighbors due to the long-range nature of the interaction potential. Another important point is that in the present work, the quasi-1D character of our system makes it different from the strict 1D FK model, that is, particles have the additional freedom to move perpendicular to the chain, which leads to a rich set of new phases.

The presence of two length scales in the FK model, that is, the interparticle distance and the periodicity of the $1 \mathrm{D}$ potential, is the reason for the complex behavior of the model. The interparticle potential favors a uniform separation between the particles, whereas the $V(x)$ tends to pin particles at the minima of the periodic potential. This competition between both interactions is often called frustration or length scales competition. If the potential $V(x)=0$, then the distance between the particles is independent of $L$ and results in a structure called a floating phase, where the equilibrium distance between particles $a$ can be an arbitrary multiple (including irrational) of the substrate periodicity $L$. Therefore, the floating phase is incommensurate except for specific values of the ratio $a / L$.

For large values of $V(x)$, we expect that particles are located at the minima of the substrate, commensurate structure, with the average spacing between particles being a rational multiple of $L$. We can observe a rich FK phase diagram in Ref. 32. Aubry showed that when the parameter $V_{0}$ is larger than a critical value, the FK system may undergo a "transition by breaking of analycity," also known as the Aubry transition. ${ }^{33}$

The minimum energy configurations of the present model system are obtained by numerical and analytical calculations. In the numerical simulations, we typically considered 100-200 particles, together with periodic boundary conditions in the unconfined direction, to mimic an infinite system. We do not consider friction in the present paper. In spite of the primary importance of friction to the motion of the particles in real systems, the ground-state configurations are not affected by it.

Notice that the substrate is defined in terms of the periodicity $L$. Comparing $L$ and $a_{0}$, we define here an initially commensurate (IC) regime when $L / a_{0}=p / q$, with $p$ and $q$ integers, and an initially noncommensurate (INC) regime of the ordered structures when the ratio $L / a_{0}$ is an irrational number. It should be emphasized that in these cases, the interparticle distance $a_{0}$ is defined in the absence of a substrate $\left(V_{0}=0\right)$. In the case $V_{0} \neq 0$, it is expected that the mean distance between particles along a given chain $a$ changes as a function of $V_{0}$, driving the system to new commensurate or noncommensurate configurations.

\section{GROUND-STATE CONFIGURATIONS}

In this section, we present the results obtained analytically and numerically for the ground-state configurations (temperature $T=0$ ). In the former, we calculate the energy per particle for different configurations as a function of the strength and periodicity of the substrate. We minimize such expressions with respect to the distances between particles. The configuration with lowest energy is the ground state. To predict which structures should be taken into account in the analytical approach, we also use molecular-dynamics simulations as a complementary tool. The numerical method can give us some hints about which structures to consider. It should be noticed that one of the drawbacks of the numerical technique is that in some cases there exists a larger number of metastable states, mainly in the limit of high densities where the system is found in a multichain structure. On the other hand, the numerical approach is the only way to obtain the ground-state configurations in incommensurate regimes, which will be analyzed in the next sections.

We show here that, depending on the periodicity of the substrate, we can tune the ground-state configuration, induce structural phase transitions, and control the number of chains. This is interesting from an experimental point of view, since the number of chains can be associated with the porosity of the system, making it a controllable filter.

The main features of the present model system can be already found in simple configurations with one and two chains $\left(V_{0}=0\right)$. For this reason, we limit ourselves to these cases, because it simplifies the physical interpretation of our results.

\section{A. Single-chain regime}

As an example, we study in this section systems with densities $n=0.5$ and $n=\sqrt{2} / 2$, which are found in the single-chain regime in the absence of the substrate $\left(V_{0}=0\right){ }^{26}$ For $n=0.5$, we consider the commensurate ratios $L / a_{0}=1$ and 2, while for $n=\sqrt{2} / 2$ we consider the noncommensurate regime with $L / a_{0}=\sqrt{2}$.

The simplest IC configuration is the trivial single-chain regime (TSC), where each particle is positioned at each minimum of the periodic potential. To exemplify such a case, we consider here the system with $n=0.5$ and $L / a_{0}=1$. In this case, the configuration remains the same for any value of $V_{0}$. According to the FK model, this is a commensurate phase. Notice that cases in which $L / a_{0}=1 / I$, where $I \geqslant 1$ is an integer, will exhibit the same behavior, since each particle is positioned exactly in a minimum of the substrate potential. On the other hand, the case $L / a_{0}=I$ is very different and the particle configuration depends strongly on $V_{0}$, as will be shown in the following.

In the IC case with $n=0.5$ and $L / a_{0}=2$, for small $V_{0}$, particles are located at the zeros of the substrate potential [see inset (I) in Fig. 2(a)]. A sufficient increase of $V_{0}$ forces the system to a new single-chain configuration in which a pair of particles is located at each minimum of the substrate [see inset (II) in Fig. 2(a)].

A further increase of $V_{0}$ pushes each pair of particles closer to each other, increasing the repulsive energy between them. For $V_{0} \approx 0.8$, a structural transition to the two-chain configuration is induced [see inset (III) of Fig. 2(a)]. In the two-chain configuration, the separation between particles $d_{x}$ in each minimum of the substrate [inset(I), Fig. 2(a)] becomes zero, which means particles become aligned along the parabolic confinement potential in each minimum $(y$ direction). In this case, the separation between chains $d_{y}$ does not change as a function of $V_{0}$, since it is ruled only by the competition between the repulsive interaction between particles and the parabolic confinement, being independent of the strength of the periodic potential. The structural transition observed here is different from the one presented in Ref. 27 , where it was shown that in the absence of a periodic potential 

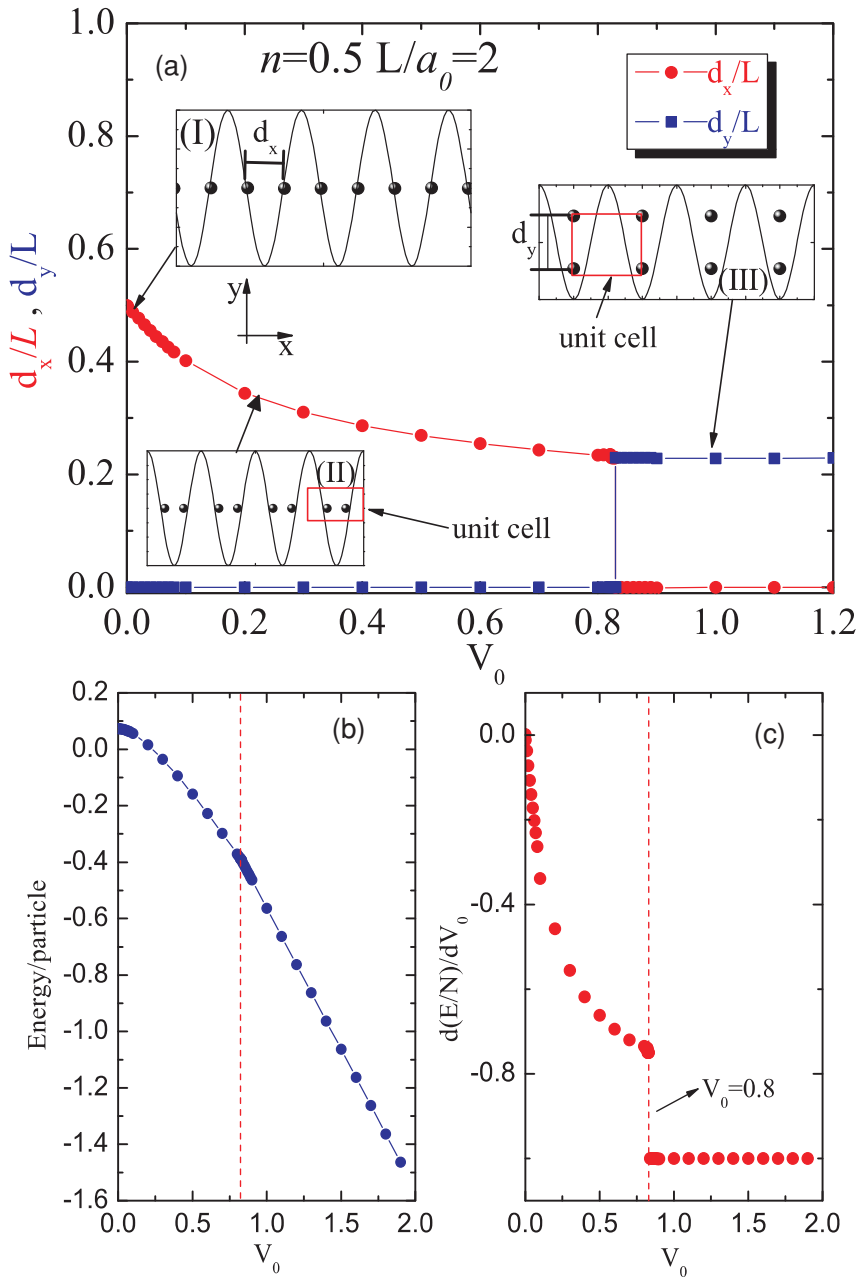

FIG. 2. (Color online) (a) Nearest-neighbor separation between particles in the $x\left(d_{x}\right)$ and $y\left(d_{y}\right)$ directions as a function of $V_{0}$ for the case $n=0.5$ and $L / a_{0}=2$. Three possible configurations are shown as insets (I), (II), and (III). (b) The energy per particle and (c) the first derivative of the energy with respect to the amplitude of the periodic potential $\left(V_{0}\right)$.

and in the presence of a parabolic confinement, the structural transition from the one- to the two-chain configuration is continuous. In the present case, the transition from the oneto the two-chain configuration is of first order. This is shown in Figs. 2(b) and 2(c), where the energy (first derivative of energy with respect to $V_{0}$ ) is continuous (discontinuous) as a function of $V_{0}$. The transition found here cannot be related to the Aubry transition since the mean particle separation is commensurate with respect to the periodic potential. Notice that in the two-chain regime, the system is reorganized in a final commensurate configuration with a new ratio $L / a=1$. The system presents a commensurate-commensurate transition between different orders of commensurability.

The structural transition observed in this case is not observed in the standard 1D FK model, however in real physical systems, even in quasi-1D chains (e.g., in biomolecules), besides the longitudinal direction (along the chain), the particles, atoms, or molecules can also move in one or two perpendicular directions, and therefore generalizations of the standard 1D FK model including transverse degrees of freedom are of great interest. The FK model can be generalized to two-dimensional versions in two different ways, leading to scalar or vector FK models. In the scalar model, ${ }^{34}$ the atoms are arranged in a 2D array, but atomic motion is still one-dimensional. In the vector FK models, ${ }^{35,36}$ the atoms can move in two dimensions, and the substrate potential is periodic in two dimensions as well.

The expression for the energy per particle, which is able to describe all phases observed in the cases $L / a_{0}=2$ and 1 in the density interval corresponding to the one- and two-chain regimes, is given by

$$
\begin{aligned}
E= & \frac{n}{2} \sum_{j} \frac{e^{-2 \kappa j / n}}{j}+\frac{n}{4} \sum_{j} \frac{e^{-\frac{2 \kappa}{n} \sqrt{\left[(j-1)+c_{x}\right]^{2}+c_{y}^{2}}}}{\sqrt{\left[(j-1)+c_{x}\right]^{2}+c_{y}^{2}}} \\
& +\frac{n}{4} \sum_{j} \frac{e^{-\frac{2 \kappa}{n} \sqrt{\left(j-c_{x}\right)^{2}+c_{y}^{2}}}}{\sqrt{\left(j-c_{x}\right)^{2}+c_{y}^{2}}}+4\left(\frac{c_{y}}{n}\right)^{2}-\cos \left(\pi c_{x}\right),
\end{aligned}
$$

where $c_{x}=d_{x} / L$ and $c_{y}=d_{y} / L$ are variational parameters.

Now we discuss the INC case with $n=\sqrt{2} / 2$ and $L / a_{0}=\sqrt{2}$. The same general behavior of previous cases can be observed here, with several structural transitions ruled by the strength of the periodic substrate $V_{0}$ (Fig. 3). An interesting feature is that for a large enough $V_{0}$, the system

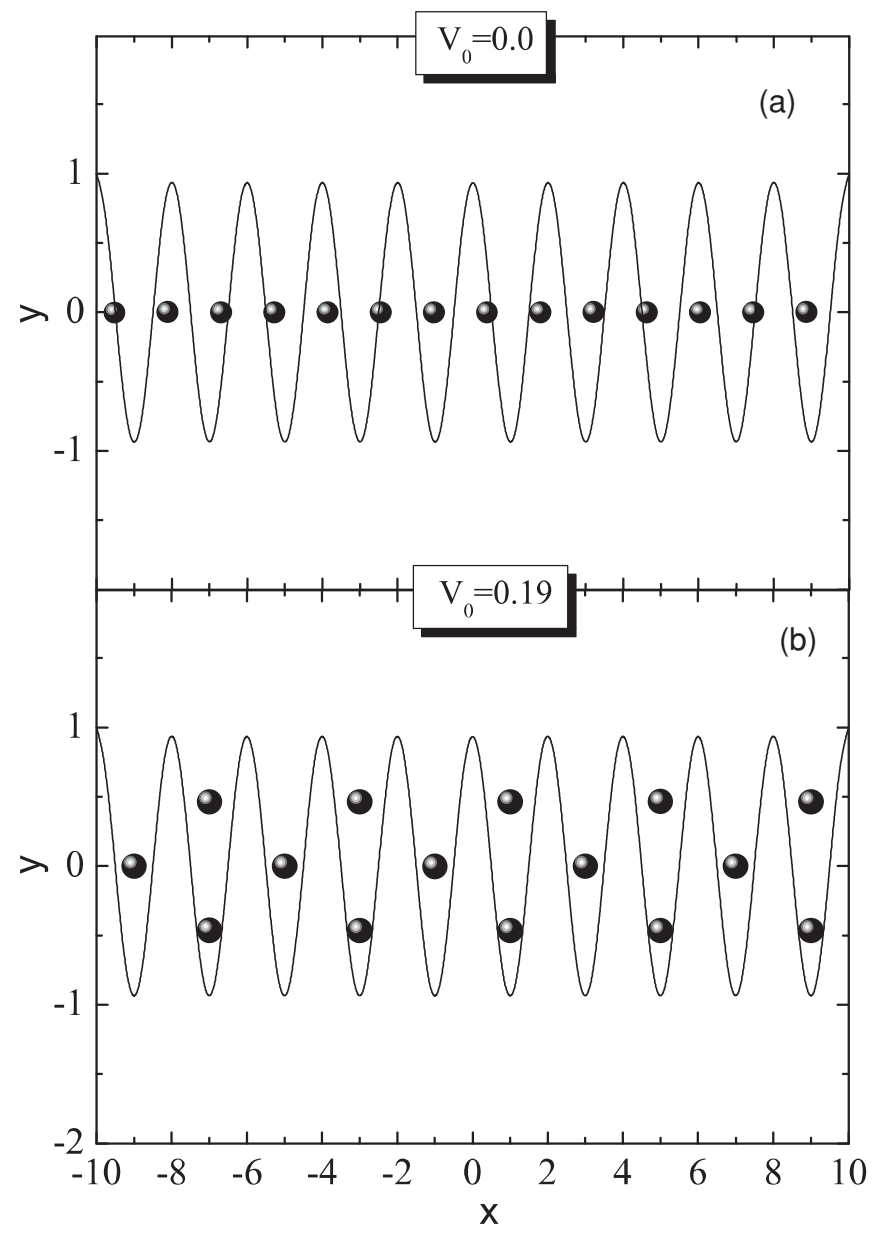

FIG. 3. Ground-state configurations for the case $n=\sqrt{2} / 2$ and $L / a_{0}=\sqrt{2}$ for (a) $V_{0}=0.17$ and (b) $V_{0}=0.19$. 
is found in a final commensurate regime with $L / a=1 / 2$, but now in the three-chain configuration with particles almost uniformly distributed over chains. In this case, we observe a noncommensurate-commensurate transition that takes place for $V_{0} \approx 0.19$. The commensurate phase is different from the one predicted by the standard FK model, where the particles are distributed randomly among the different substrate minima. For a more comprehensive discussion about chaotic phases, see, for instance, Ref. 32.

\section{B. Two-chain regime}

In this section, we consider as an example the system with $n=1.0$, where a two-chain configuration is found as the ground state for $V_{0}=0$. Different from what was observed in the single-chain configuration $(n=0.5)$, when $L / a_{0}=1$ the system is always found in the two-chain configuration, but the internal structure depends on $V_{0}$. This is shown in Fig. 4(a), where the relevant internal distances [see Fig. 4(b)] for the ground-state configuration are presented as a function of $V_{0}$.

For $V_{0} \approx 0.16$, the system changes from a staggered $\left(d_{x} \neq\right.$ $0)$ to an aligned $\left(d_{x}=0\right)$ two-chain configuration through a second-order (continuous) structural transition, characterized by a continuity (discontinuity) in the first (second) derivative of the energy with respect to $V_{0}$ [Fig. 4(d)]. Notice that the distances $\left(d_{x}, d_{y}\right)$ change continuously with $V_{0}$, while in the case with $n=0.5, L / a=2.0$ they exhibit a jump, which is another way to find out the order of the structural phase transition. The type of transition found in this case is not an Aubry transition since the mean particle separation is commensurate with respect to the substrate. These kinds of particle configurations found here are also observed in the quasi-1D-FK model (see, e.g., Ref. 37 for a detailed discussion on transitions in 2D FK models).

Next, we consider the more complex case with $n=1.0$ and $L / a_{0}=2$. Several interesting configurations are observed with increasing $V_{0}$, as shown in Fig. 5. Initially, particles move along the $x$ direction toward the minima of the periodic potential. At the same time, each chain starts to break up into two chains [Fig. 5(c)]. The structural transition found in this case is of second order.

For larger $V_{0}$, the two inner chains move toward each other [see Fig. 5(d)] and merge into a single chain in the center [see Figs. 5(d)-5(f)]. The particles in the outer chains move toward the minimum of the periodic potential [see Figs. 5(d)$5(f)]$. With further increase of $V_{0}$, the pair of particles in the middle chain are pushed closer to each other and finally form a row of four particles along the $y$ direction and positioned in each minimum of the periodic potential [see Figs. 5(g) and 5(h)]. The configurations presented in Fig. 5 indicate a tunable porosity of the system as a function of $V_{0}$. This is a very interesting and useful feature for a filter or sieve, as pointed out in Refs. 38 and 39, where superparamagnetic colloidal particles were self-assembled in chainlike structures and used for the separation of DNA molecules.

The movement of the different particles in the $x$ and $y$ directions as a function of $V_{0}$ is summarized in Fig. 6, where structural transitions are indicated by vertical dashed lines. In the $x$ direction, we limited our analysis to the four particles around the minimum located at $x=2$. Three second-order
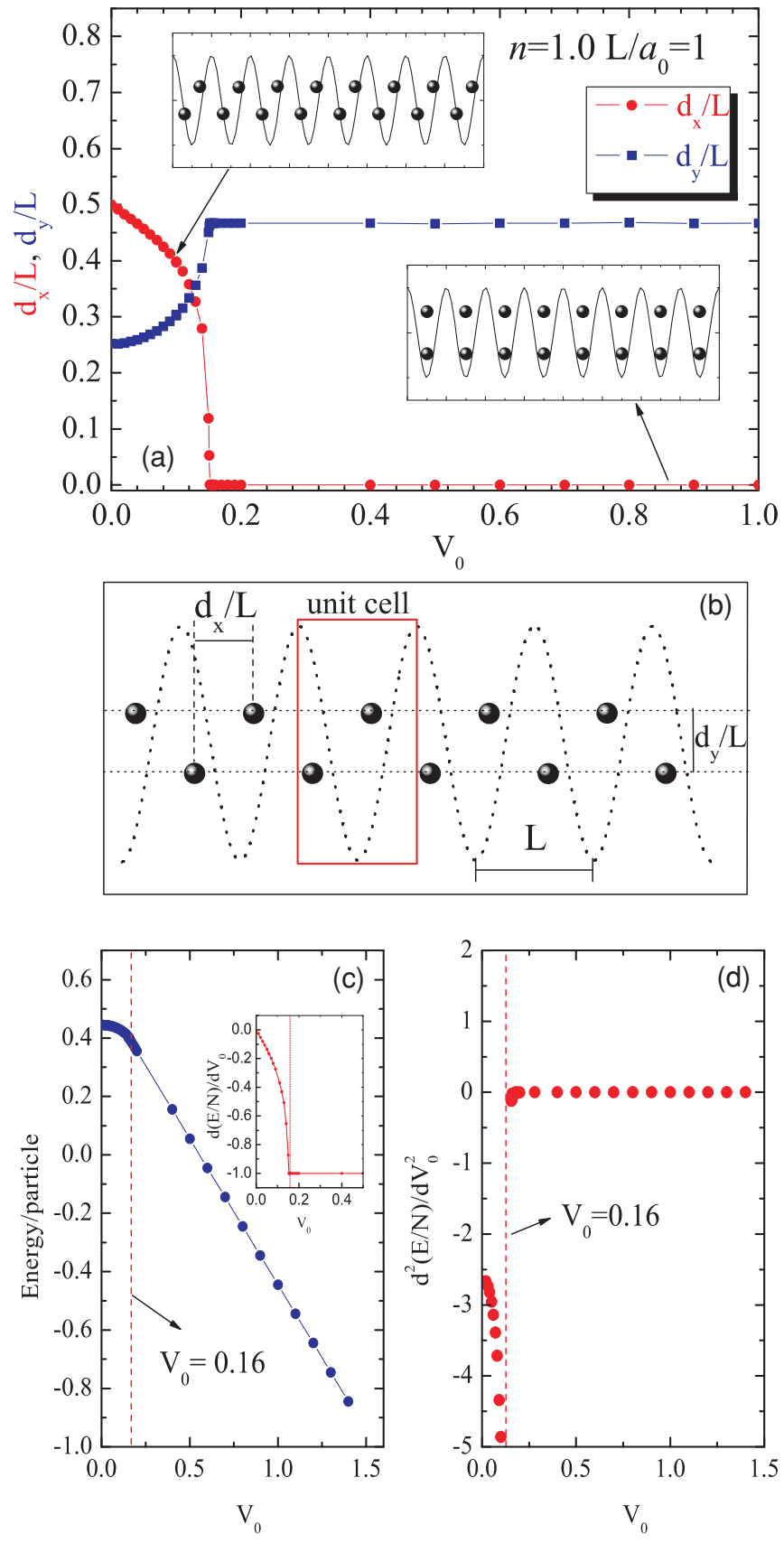

FIG. 4. (Color online) (a) Interparticle separation as a function of $V_{0}$ for $n=1.0$ and $L / a_{0}=1$. (b) A sketch of the two-chain configuration with the distances $d_{x}$ and $d_{y}$ indicated. (c) The energy per particle and (d) the second derivative of the energy with respect to the intensity of the periodic substrate.

structural transitions are observed as a function of $V_{0}$, and the number of chains varies in the following sequence: $2 \rightarrow 4 \rightarrow$ $3 \rightarrow 4 \rightarrow 4$.

Here, we observe that particles initially disposed in a zigzag structure go to different configurations. These kinds of particle distributions are also observed in the FK model (see Ref. 40).

Finally, we consider as an example the case with $n=1.5$ and $L / a_{0}=1.5$, which exhibits a very interesting feature not found in the previous cases. Here, we show that the 

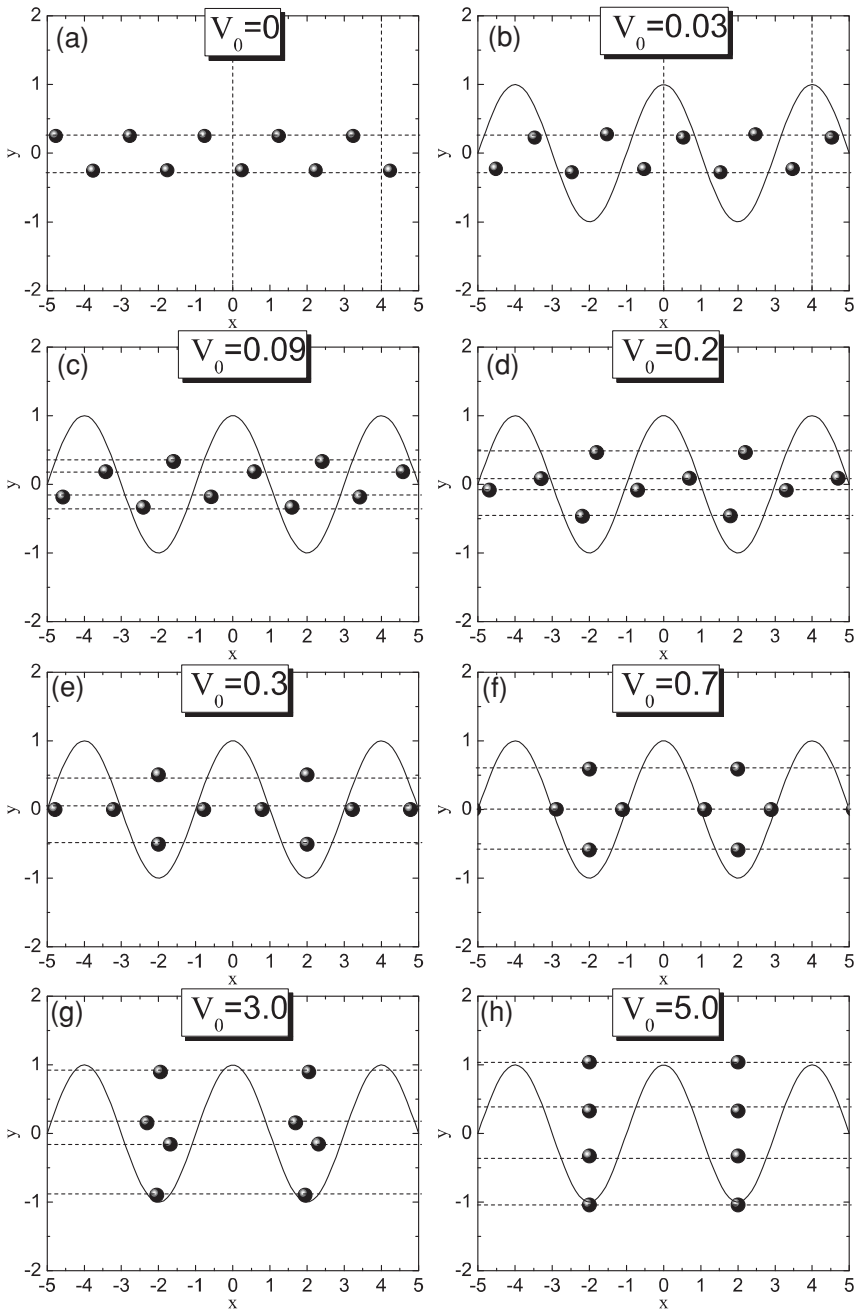

FIG. 5. Ground-state configurations for the case $n=1.0$ and $L / a_{0}=2$ for different values of $V_{0}$. The vertical dashed lines in (b) delimit the region where we analyze our system.

number of particles in the unit cell can be changed as a function of $V_{0}$. Initially $\left(V_{0}=0\right)$, the system is arranged in two chains [Fig. 7(a)], which are displaced with respect to each other over half the interparticle distance in each chain. There are two particles per unit cell, which characterize an IC configuration.

When $V_{0}$ increases, the system transits to a four-chain configuration through a second-order structural transition, with the outer chains having twice as many particles as the inner chains [Fig. 7(b)]. Alternatively, we can also view this configuration as two chains of triangles, as indicated in the shadowed region in Fig. 7(b). In this case, $d_{2}\left(=d_{5}\right)>d_{3}\left(=d_{4}\right)$ and the length of the unit cell is $d_{1}=d_{2}+d_{3}$. There are six particles in the unit cell, which is twice the number of particles in the unit cell in the case $V_{0}=0$.

With a further increase of $V_{0}$, the $y$ distance $d_{6}$ between the internal chains goes to zero and the system changes to the three-chain configuration [Fig. 7(c)] with the same number of particles in each one, and the central chain is shifted by $a / 2$ along the $x$ direction with respect to the outer chains, which are aligned along the $y$ direction. Notice that in this case there

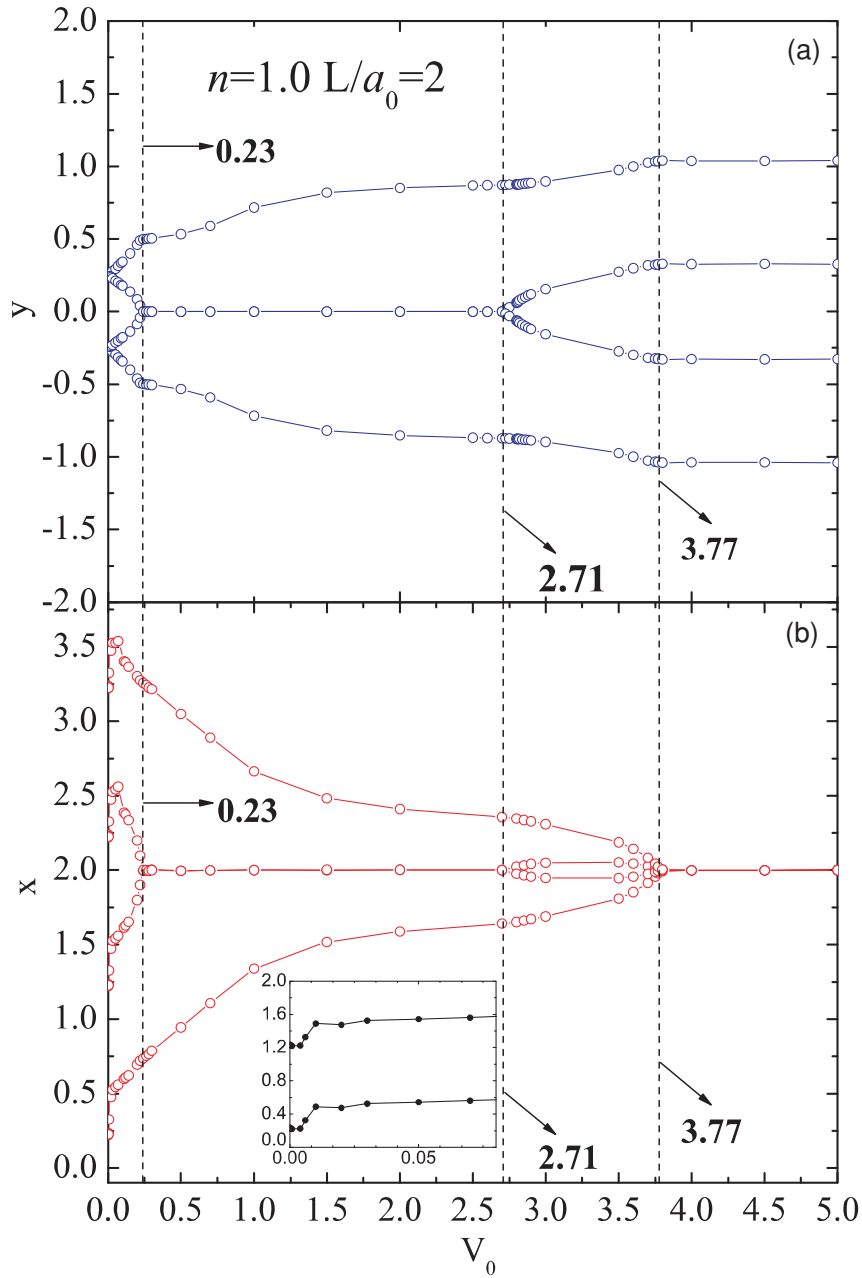

FIG. 6. (Color online) (a) The lateral $y$ position of chains for the case $n=1.0$ and $L / a_{0}=2$. The vertical dotted lines represent the values of $V_{0}$ where structural transitions occur. (b) The particle position in the $x$ direction as a function of $V_{0}$. The inset shows the small $V_{0}$ region.

are only three particles per unit cell. This is interesting since the number of normal modes is now half that observed for the configuration presented in Fig. 7(b), where the number of particles in the unit cell is six. The reduction of the allowed excitation modes is controlled by the strength of the periodic potential, and this can be used as an important feature for possible applications in phononics. For $V_{0} \geqslant 0.5$, particles in different chains are all aligned along the $y$ direction and located in each minimum of the periodic substrate [Fig. 7(d)]. The trajectories of the different particles in the channel as a function of $V_{0}$ is visualized in Fig. 8 .

Again, for $V_{0} \geqslant 0.5$ the relation between the periodicity of the substrate and the distance between particles is different from the case $V_{0}=0$. This is interesting since we can change the commensurability of the system by changing only the strength of the substrate potential.

As presented in Figs. 2(a), 4(a), and 5(h), for a critical value of $V_{0}$ the present model system is found in the special configuration where particles become aligned along the confinement direction. Such a $y$-aligned configuration (YAC) 

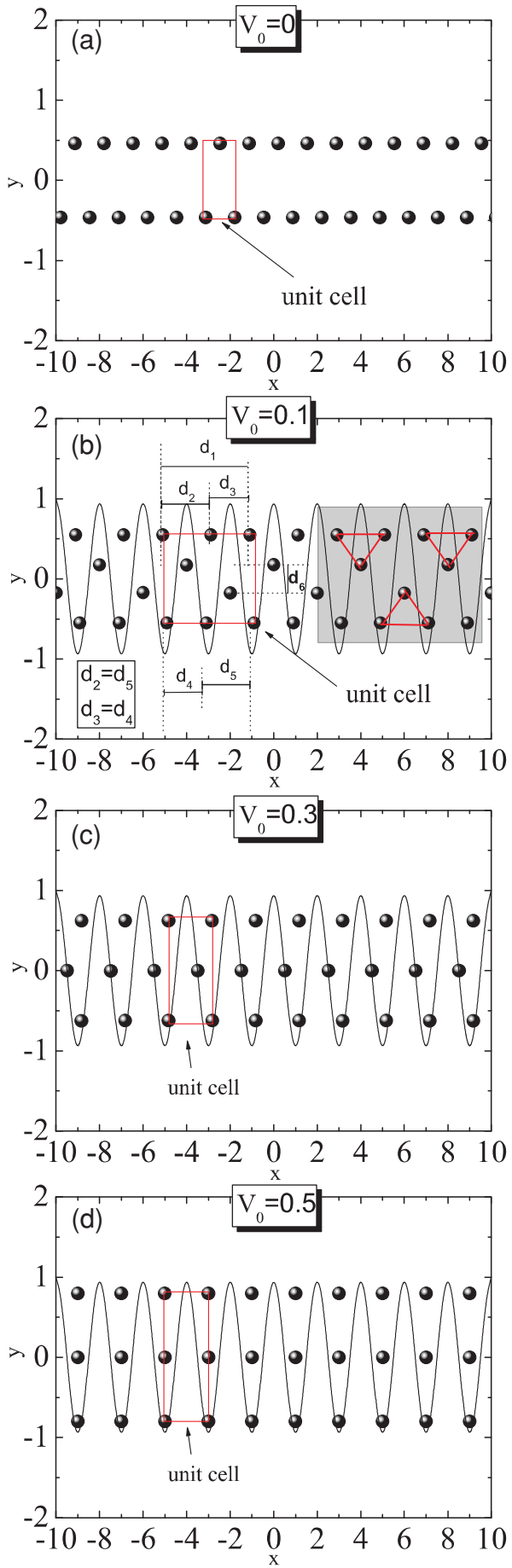

FIG. 7. (Color online) Ground-state configurations for different values of $V_{0}$ for $n=1.5$ and $L / a_{0}=1.5$. In (b) the relevant distances that define the configuration are presented.

occurs if the condition $L / a_{0}=p$, where $p$ is an integer $(\geqslant 1)$, is satisfied. In this case, if $N$ is the number of chains of the initial structure $\left(V_{0}=0\right)$, then we find that the number of particles aligned along the $y$ direction in each minimum of the substrate potential is $N p$, which is also the number of chains. The critical value of $V_{0}$ for which the YAC phase is induced $\left(V_{c}\right)$ is obtained by adding the interaction energy between particles and the confinement energy. A general expression for

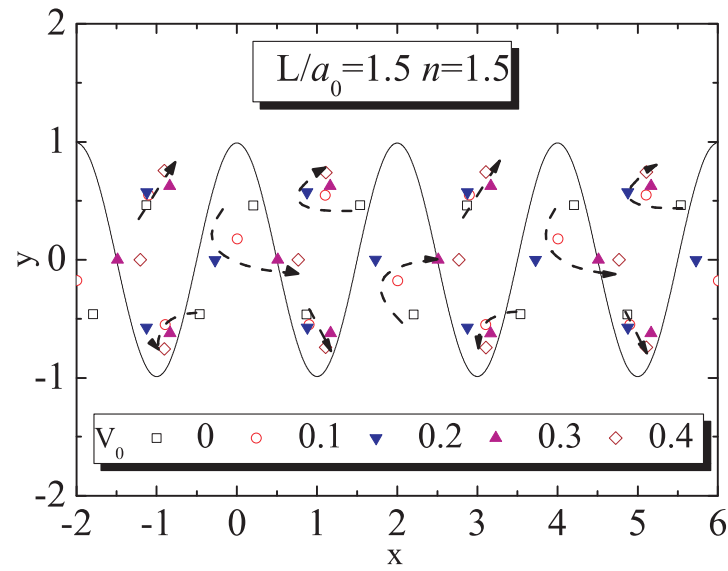

FIG. 8. (Color online) Displacement of the particles for different values of $V_{0}$ near the potential minimum for the case $n=1.5$ and $L / a_{0}=1.5$.

the YAC is given by

$$
\begin{aligned}
V_{c}= & \frac{n}{N p} \sum_{j} \frac{e^{-k N p j / n}}{j}+\frac{2 n}{N^{2} p^{2}} \sum_{q=1}^{N p-1} \sum_{l=q+1}^{N p} \sum_{j} \\
& \times \frac{e^{-k N p / n \sqrt{j^{2}+(p-q)^{2} c^{2}}}}{\sqrt{j^{2}+(p-q)^{2} c^{2}}}+\frac{n}{N^{2} p^{2}} \sum_{q=1}^{N p-1} \sum_{l=q+1}^{N p} \\
& \times \frac{e^{-k(l-q) c N p / n}}{(l-q) c}+\frac{2 c^{2} N p}{n^{2}} \sum_{l=1}^{N p / 2}(l-1 / 2)^{2},
\end{aligned}
$$

in the case in which $N p$ is even, and

$$
\begin{aligned}
V_{c}= & \frac{n}{N p} \sum_{j} \frac{e^{-k N p j / n}}{j}+\frac{2 n}{N^{2} p^{2}} \sum_{q=1}^{N p-1} \sum_{l=q+1}^{N p} \sum_{j} \\
& \times \frac{e^{-k N p / n \sqrt{j^{2}+(p-q)^{2} c^{2}}}}{\sqrt{j^{2}+(p-q)^{2} c^{2}}}+\frac{n}{N^{2} p^{2}} \sum_{q=1}^{N p-1} \sum_{l=q+1}^{N p} \\
& \times \frac{e^{-k(l-q) c N p / n}}{(l-q) c}+\frac{2 c^{2} N p}{n^{2}} \sum_{l=1}^{N p / 2} l^{2}
\end{aligned}
$$

if $N p$ is an odd number.

The critical value of $V_{0}$ and the separation between particles in each minimum $d$ in the YAC phase at $V_{c}$ are presented in Fig. 9. A sketch of the configuration in each minimum of the periodic substrate with all relevant parameters is also shown as the inset in Fig. 9(b). A power-law dependence of $V_{c}$ and $d / L$ on the density is found, with the product $V_{c} d / L \approx$ const.

\section{PHONON SPECTRUM}

Next, we analyze the $V_{0}$ dependence of the normal-mode spectrum. We follow the standard harmonic approximation and take into account the periodicity of the system in the unconfined direction ( $x$ axis).

The number of particles in the unit cell and the number of degrees of freedom per unit cell determines the number of branches in the phonon spectrum. If $l$ is the number of particles 

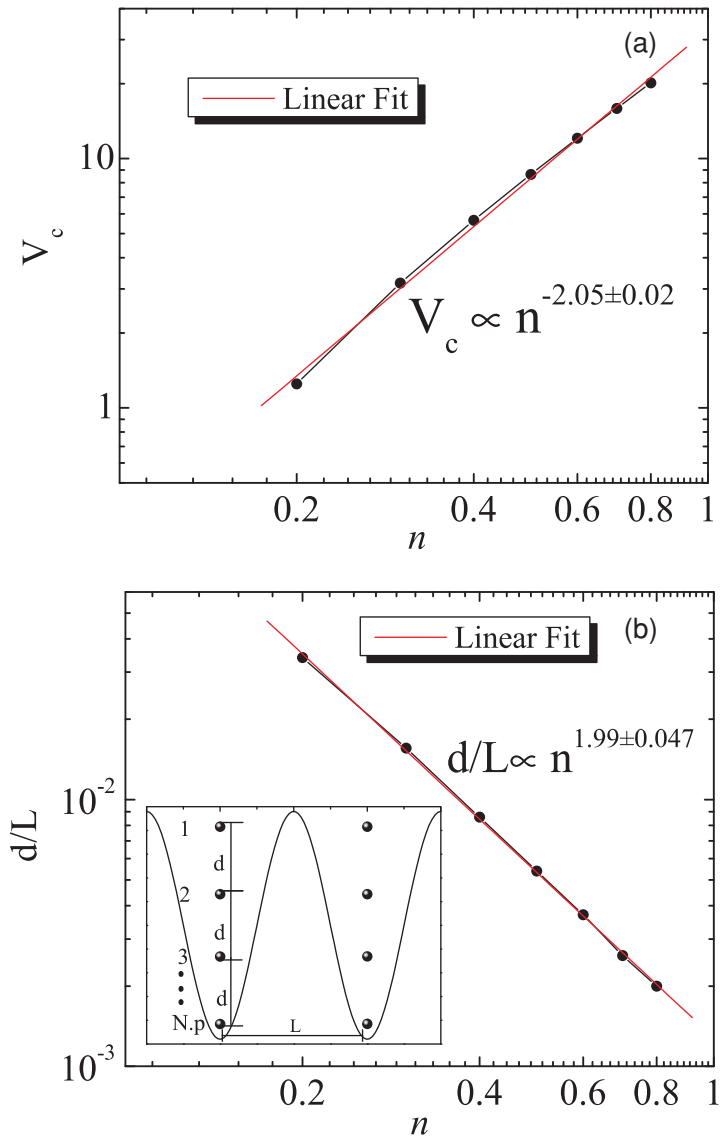

FIG. 9. (Color online) (a) Critical value of $V_{0}$ as function of density for the YAC phase. (b) The distance between the particles along the $y$ direction at $V_{0, c}$ as a function of the density in the YAC phase. The inset shows a sketch of the general ground-state configuration with all relevant parameters. The red line in both figures is a linear fit.

per unit cell, there will be $2 l$ branches in the phonon spectrum, from which half of those branches corresponds to oscillations along the chain, that is, longitudinal modes, while the others are associated with vibrations along the confinement direction ( $y$ axis, transverse modes). If particles in the unit cell oscillate in-phase, the mode is dominantly acoustical, while the opposite out-of-phase oscillation corresponds to an optical mode. In general, a normal mode can be classified in one of the following classes: longitudinal optical (LO), longitudinal acoustical (LA), transverse optical (TO), or transverse acoustical (TA).

In the harmonic approximation, the normal modes are obtained by solving the system of equations

$$
\left(\omega^{2} \delta_{\mu \nu, i j}-D_{\mu \nu, i j}\right) Q_{\nu, j}=0
$$

where $Q_{v, j}$ is the displacement of particle $j$ from its equilibrium position in the $v$ direction, $\mu$ and $v$ refer to the spatial coordinates $x$ and $y, \delta_{\mu \nu, i j}$ is the unit matrix, and $D_{\mu \nu, i j}$ is the dynamical matrix, defined by

$$
D_{\mu \nu, i j}=\frac{1}{m} \sum_{u} \phi_{\mu, v}(u) e^{-i u q a}
$$

where $u$ is an integer assigned to each unit cell. The force constants are given by

$$
\phi_{\mu, v}(u)=\partial_{\mu} \partial_{\nu} \frac{\exp \left[-\kappa \sqrt{\left(x-x^{\prime}\right)^{2}+\left(y-y^{\prime}\right)^{2}}\right]}{\sqrt{\left(x-x^{\prime}\right)^{2}+\left(y-y^{\prime}\right)^{2}}},
$$

with $\left(x-x^{\prime}\right)$ the distance between particles along the $x$ axis and $\left(y-y^{\prime}\right)$ the interchain distance with $(x, y)$ and $\left(x^{\prime}, y^{\prime}\right)$ the equilibrium positions of the particles in the unit cell, and

$$
\phi_{\mu, v}(u=0)=-\sum_{u \neq 0} \phi_{\mu, v}(u) .
$$

The phonon frequency is given in units of $\omega_{0} / \sqrt{2}$. As an example, the complete dynamical matrix for the one- and twochain regimes is given in the Appendix.

The frequencies for the one-chain configuration in the case $V_{0}=0$ are given by $\omega_{l}=\sqrt{A_{1}}$ for the acoustical branch and $\omega_{t}=\sqrt{1+A_{2}}$ for the optical branch, where $A_{1}$ and $A_{2}$ are given in the Appendix.

The frequencies for the one- and two-chain configurations when $V_{0} \neq 0$ are given by

$\omega_{l}=\sqrt{\frac{1}{4}\left(B_{1}+B_{3} \pm \sqrt{B_{1}^{2}+4 B_{5} B_{7}-2 B_{1} B_{3}+B_{3}^{2}}+\mathrm{sub}\right)}$

for the longitudinal modes, and by

$\omega_{t}=\frac{1}{2} \sqrt{4+B_{2}+B_{4} \pm \sqrt{B_{2}^{2}+4 B_{6} B_{8}-2 B_{2} B_{4}+B_{4}^{2}}}$

for the transverse modes. The expressions for $B_{1}, B_{2}, \ldots, B_{8}$ are given in the Appendix. Here, sub $=8 V_{0} \pi^{2} \cos \left(\pi c_{x}\right)$ is the term related to the substrate. The wave number $k$ for the oneand the two-chain regimes is in units of $2 \pi / L$, where $L$ is also the length of the unit cell in the $x$ direction.

In Fig. 10(a), the phonon spectrum for the one-chain configuration is presented for different values of $V_{0}$ and fixed density $n=0.5$ and $L / a_{0}=1$. In this case, there is one particle per unit cell located in each minimum of the substrate resulting only in one longitudinal mode and one transverse mode. The frequency of the longitudinal mode increases with increasing $V_{0}$, and there is a gap opening at $k=0$. The reason is that the periodic potential acts locally

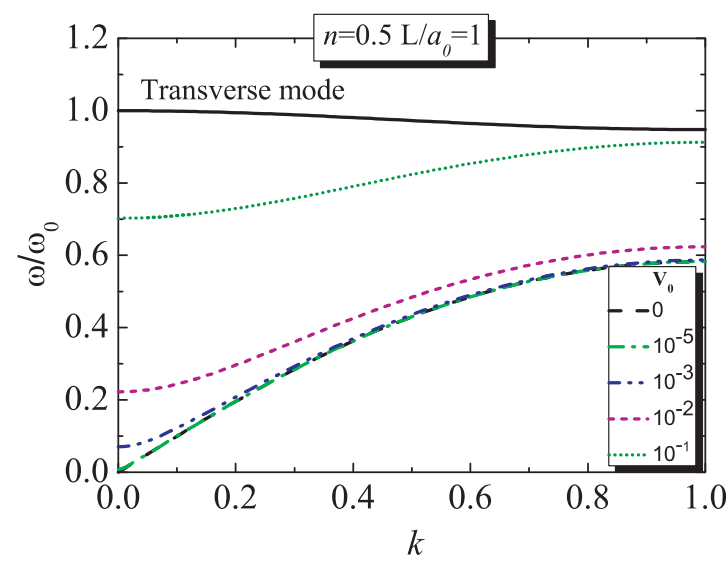

FIG. 10. (Color online) The phonon spectrum for different values of $V_{0}$ in the case $n=0.5$ and $L / a_{0}=1$. 
as a parabolic confinement potential $V(x) \simeq V_{0} \frac{2 \pi^{2}}{L^{2}} x^{2}$ with frequency $\omega=\sqrt{V_{0} / m} \frac{2 \pi}{L}$. The $k=0$ gap corresponds to this frequency. The transverse mode corresponds to particle oscillations in the $y$ direction and is therefore practically independent of $V_{0}$.

In Fig. 11, the dispersion curves for $n=0.5$ and $L / a_{0}=2$ are presented for different values of $V_{0}$. As observed in Fig. 2, the presence of the substrate $\left(V_{0} \neq 0\right)$ modifies the number of particles in the unit cell so that the number of branches of the phonon spectrum is increased as compared to the case $V_{0}=0$ [Fig. 11(a)]. For $V_{0} \neq 0$, there are two particles per unit cell and consequently four branches in the phonon spectrum. As $V_{0}$ increases, the frequency of the LA mode also increases, which can be explained keeping in mind that for low values of $V_{0}$, there is a small electrostatic repulsion between neighboring particles, so that particles oscillate horizontally without major difficulties. The opposite behavior is found for the TO mode, that is, it decreases with increasing $V_{0}$. The distance between adjacent particles in the same substrate minimum becomes smaller, and the repulsive force between them increases and acts as a retarding force.

The LO mode has a rather different behavior as compared to the TO mode, that is, there is a hardening of its frequency when $V_{0}$ increases, which is a consequence of the larger repulsion due to the closer proximity between particles. For a sufficiently strong $V_{0} \approx 0.2$ [Fig. 11(c)], the normal-mode spectrum becomes discrete, that is, frequencies become independent of $k$, which means the group velocity is zero and the modes are localized. In this case, the oscillations of the particles in each minimum of the substrate become independent and there is no wave propagation along the channel. A structural phase transition to the two-chain configuration, with particles aligned along the $y$ direction (YAC phase) in each minimum of the substrate (Fig. 2), occurs for $V_{0} \geqslant 0.8$. The longitudinal mode frequency becomes much harder than the transverse ones due to the strong confinement imposed by the periodic substrate.

Now we discuss the dispersion curves for the system with density $n=1.0$ and $L / a_{0}=1$ (Fig. 12). As presented in Fig. 4 , particles remains in the two-chain configuration for all $V_{0}$ with changes only in the internal structure. Again, the substrate potential induces gaps in the normal-mode frequencies as presented in Fig. 12. The TA, TO, LA, and LO modes increase with increasing $V_{0}$.

In the case of the LA mode, for low values of $V_{0}$, particles are not aligned, having more freedom to oscillate in the horizontal direction. When $V_{0}$ increases, the electrostatic force becomes larger (particles are now aligned), making oscillations along the channel more difficult.

The LO mode also increases with increasing $V_{0}$. This is a consequence of the strength of the substrate potential, which traps particles in their equilibrium positions, reducing the out-of-phase oscillations of the particles. The TO frequency increases slightly, since the out-of-phase motion is more difficult to occur. The TA frequency branch is almost independent of $V_{0}$ because it corresponds to oscillations in the $y$ direction and is therefore determined by the harmonic confinement potential with frequency $\omega_{0}$.

For the YAC phase, $V_{0}>0.16$, the normal-mode spectrum becomes discrete [Fig. 12(d)]. The modes are almost constant
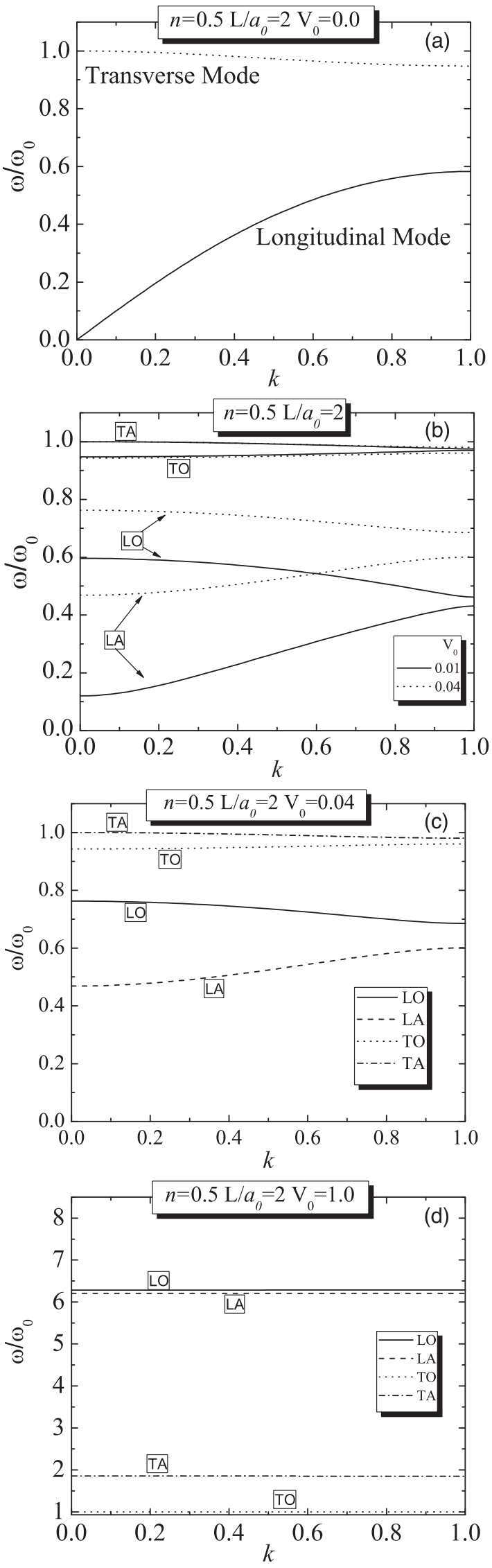

FIG. 11. The phonon spectrum for different values of $V_{0}$ in the case $n=0.5$ and $L / a_{0}=2$. 

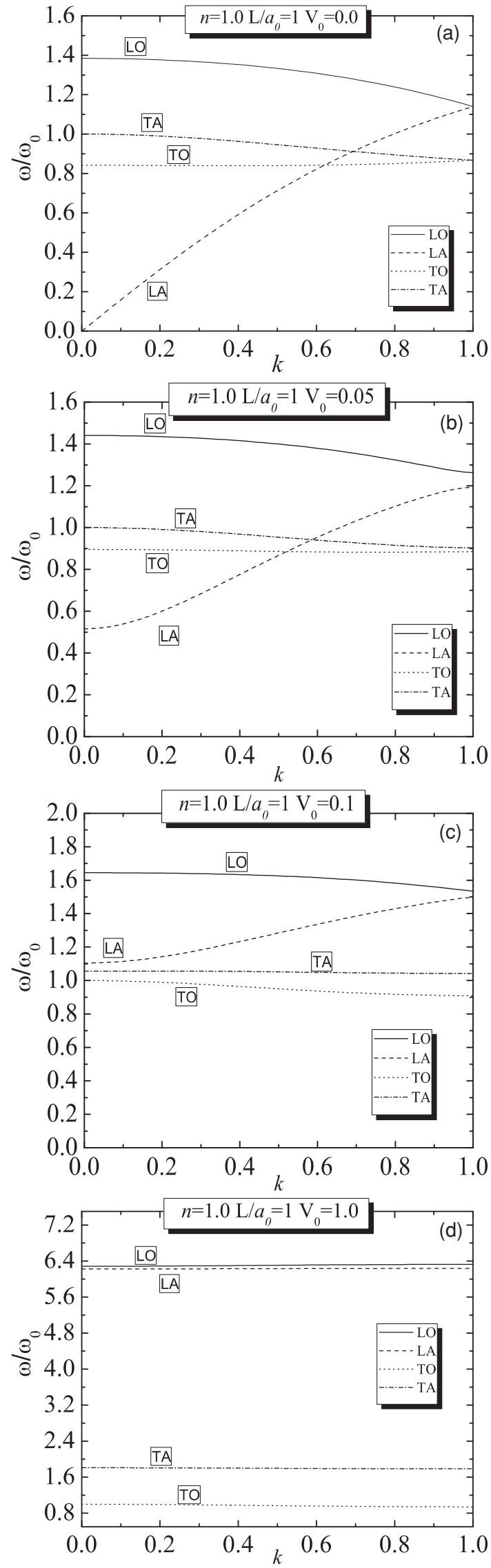

FIG. 12. The phonon spectrum for different values of $V_{0}$ in the case $n=1$ and $L / a_{0}=1$. due to the strong confinement potential imposed by the substrate and the harmonic trap. ${ }^{41-43}$

\section{CONCLUSIONS}

We investigated the structural and dynamical properties of a two-dimensional system of repulsive particles confined by a parabolic channel and submitted to a one-dimensional periodic potential (substrate). The ground-state configurations $(T=$ 0 ) were obtained analytically and numerically, where for the latter we used molecular-dynamics simulations. The phonon spectrum were also calculated analytically for the one- and two-chain configurations within the harmonic approximation.

The main features of the structure and normal-mode spectrum were studied (for different densities) as a function of the periodicity $(L)$ and strength $\left(V_{0}\right)$ of the substrate, which are experimentally tunable parameters in systems such as, for example, colloids in the presence of a periodic light field composed of two interfering laser beams. An interesting set of ground-state configurations with controllable porosity was observed mainly as a function of $V_{0}$ through several first- or second-order structural transitions. The structures are mainly ruled by the fact that particles tend to go to the minima of the periodic substrate, modifying the symmetry of the ordered structures. However, for small $V_{0}$, the interparticle repulsive interaction dominates and particles can be found over all possible positions in the periodic potential, including regions near to the maxima. For large $V_{0}$, particles are more and more attracted to the wells of the periodic potential.

For some specific cases, we found structural transitions where the number of particles in the unit cell of the periodic system is changed, implying, for example, a different number of branches in the phonon spectrum, which is an interesting aspect of the dynamical behavior of the system, and which may be useful for applications in phononics.

The normal-mode frequencies depend on the linear density of the system, periodicity, and strength of the periodic substrate. We observed gaps in the phonon spectrum, which indicate that there are frequencies blocked by the crystal. For $V_{0}$ beyond a critical value and for specific values of the ratio $L / a_{0}$, the system is found in a special configuration where particles are aligned in each minimum of the periodic substrate along the $y$ direction (YAC phase). For such a configuration, the normal-mode frequencies become independent of the wave vector, which means there is no wave propagation along the channel since the group velocity is zero. The modes are localized and the phonon spectrum becomes discrete. In this case, the oscillations of particles in different minima of the substrate are independent. In the YAC regime, the discrete normal-mode spectrum indicates that the system can be seen as a set of independent clusters.

Our model system of Yukawa particles can be realized experimentally using a dusty plasma $^{15,16}$ or colloidal systems. ${ }^{17,18}$ In the former, the dust particles are confined to a two-dimensional layer through a combination of gravitational and electrical forces. By microstructuring a channel in the bottom electrode of the discharge, it is possible to laterally confine the dust particles, as was realized in Refs. 19-23. The strength of the 1D confinement potential can be varied by the width of the channel or the potential on the bottom 
electrode. When the width of the channel is microstructured into an oscillating function along the channel, it will result in a periodic potential along the channel.

In the latter, one can also confine charged colloids, which move in a liquid environment containing counterions, into microchannels as recently realized experimentally in Ref. 24. In this case, the intercolloid interaction can be modeled by a screened Coulomb interaction and the confinement potential is a hard-wall potential. By changing the depth profile of the microchannel, it was shown in Ref. 25 that the confinement potential can be tuned into a harmonic potential. Microstructuring the width of the channel into an oscillating function along the channel will result in an additional periodic potential along the channel.

Here, we conclude that the structural and dynamical properties of one-dimensional systems subject to periodic substrates can be tuned by the strength of the periodic potential. The present results indicate a rich set of configurations in which colloids can be manipulated in the presence of external fields. The tunable phonon spectrum is a very interesting feature for phononic or photonic ${ }^{6}$ band-gap materials.

\section{ACKNOWLEDGMENTS}

This work was supported by the Brazilian agencies $\mathrm{CNPq}$ and FUNCAP (PRONEX-Grant), and the bilateral projects between Flanders and Brazil and the Flemish Science Foundation (FWO-Vl) and CNPq.

\section{APPENDIX}

The matrix $\omega^{2} \mathbf{I}-\mathbf{D}$ (where $\mathbf{I}$ is the unit matrix and $\mathbf{D}$ is the dynamical matrix) is used in the calculation of the normal modes for the one- and two-chain configurations. The dynamical matrix for the one-chain configuration when $V_{0}=0$ is

$$
\left[\begin{array}{ll}
\omega^{2}-A_{1} & 0 \\
0 & \left(\omega^{2}-\omega_{0}^{2}\right)-A_{2}
\end{array}\right],
$$

where the quantities $A_{1}$ and $A_{2}$ are given by

$$
\begin{aligned}
A_{1}= & \sum_{j=1}^{\infty} n^{3} \frac{e^{-\kappa j / n}}{j^{3}}\left[2+\frac{2 \kappa j}{n}+\frac{\kappa^{2} j^{2}}{n^{2}}\right][1-\cos (k \pi j)] \\
& +V_{0} \pi n^{2} \cos (\pi j), \\
A_{2}= & \sum_{j=1}^{\infty} n^{3} \frac{e^{-\kappa j / n}}{j^{3}}\left[1+\frac{\kappa j}{n}\right][1-\cos (k \pi j)],
\end{aligned}
$$

where $j$ is an integer.

The dimensionless wave number $k$ is in units of $2 \pi / L$. The dynamical matrix to one-chain $V_{0} \neq 0$ and two-chain configuration is

$$
\left[\begin{array}{llll}
\omega^{2}-B_{1}-\operatorname{sub} & 0 & -B_{5} & 0 \\
0 & \Delta \omega^{2}-B_{2} & 0 & -B_{6} \\
-B_{7} & 0 & \omega^{2}-B_{3}-\mathrm{sub} & 0 \\
0 & -B_{8} & 0 & \Delta \omega^{2}-B_{4}
\end{array}\right],
$$

where $\Delta \omega^{2}=\omega^{2}-\omega_{0}^{2}$. The quantities $B_{1}, B_{2}, B_{3}, B_{4}, B_{5}$, and $B_{6}$ are given by

$$
\begin{aligned}
& B_{1}=\sum_{j=1}^{\infty} n^{3} \frac{e^{-2 \kappa r / n}}{(2 r)^{3}}\left[\left(j-c_{x}\right)^{2}\left(\frac{3}{r^{2}}+\frac{6 \kappa}{n r}+\frac{4 \kappa^{2}}{n^{2}}\right)\right. \\
& \left.-\left(1+\frac{2 \kappa r}{n}\right)\right]+\sum_{j=1}^{\infty} n^{3} \frac{e^{-2 \kappa j / n}}{(2 j)^{3}} \\
& \times\left[2+\frac{4 \kappa j}{n}+\frac{(2 \kappa j)^{2}}{n^{2}}\right][1-\exp (i k j L)], \\
& B_{2}=\sum_{j=1}^{\infty} n^{3} \frac{e^{-2 \kappa r / n}}{(2 r)^{3}}\left[\frac{3 c_{y}^{2}}{r^{2}}+\frac{4 \kappa^{2} c_{y}^{2}}{n^{2}}+\frac{6 \kappa c_{y}^{2}}{n r}-\left(1+\frac{\kappa r}{n}\right)\right] \\
& -\sum_{j=1}^{\infty} n^{3} \frac{e^{-2 \kappa j / n}}{(2 j)^{3}}\left[1+\frac{2 \kappa j}{n}\right][1-\exp (i k j L)], \\
& B_{3}=\sum_{j=1}^{\infty} n^{3} \frac{e^{-2 \kappa r_{1} / n}}{(2 r)^{3}}\left[\left(j-1+c_{x}\right)^{2}\left(\frac{3}{r^{2}}+\frac{6 \kappa}{n r}+\frac{4 \kappa^{2}}{n^{2}}\right)\right. \\
& \left.-\left(1+\frac{2 \kappa r_{1}}{n}\right)\right]+\sum_{j=1}^{\infty} n^{3} \frac{e^{-2 \kappa j / n}}{(2 j)^{3}} \\
& \times\left[2+\frac{4 \kappa j}{n}+\frac{(2 \kappa j)^{2}}{n^{2}}\right][1-\exp (i k j L)], \\
& B_{4}=\sum_{j=1}^{\infty} n^{3} \frac{e^{-2 \kappa r_{1} / n}}{\left(2 r_{1}\right)^{3}}\left[\frac{3 c_{y}^{2}}{r_{1}^{2}}+\frac{4 \kappa^{2} c_{y}^{2}}{n^{2}}+\frac{6 \kappa c_{y}^{2}}{n r_{1}}-\left(1+\frac{\kappa r_{1}}{n}\right)\right] \\
& -\sum_{j=1}^{\infty} n^{3} \frac{e^{-2 \kappa j / n}}{(2 j)^{3}}\left[1+\frac{2 \kappa j}{n}\right][1-\exp (i k j L)], \\
& B_{5}=\sum_{j=1}^{\infty} n^{3} \frac{e^{-2 \kappa r / n}}{(2 r)^{3}}\left[\left(j-c_{x}\right)^{2}\left(\frac{3}{r^{2}}+\frac{6 \kappa}{n r}+\frac{4 \kappa^{2}}{n^{2}}\right)\right. \\
& \left.-\left(1+\frac{2 \kappa r}{n}\right)\right]\left\{\exp \left[i k L\left(j-c_{x}\right)\right]\right\}, \\
& B_{6}=\sum_{j=1}^{\infty} n^{3} \frac{e^{-2 \kappa r / n}}{(2 r)^{3}}\left[\frac{3 c_{y}^{2}}{r^{2}}+\frac{4 \kappa^{2} c_{y}^{2}}{n^{2}}+\frac{6 \kappa c_{y}^{2}}{n r}\right. \\
& \left.-\left(1+\frac{\kappa r}{n}\right)\right]\left\{\exp \left[i k L\left(j-c_{x}\right)\right]\right\}, \\
& B_{7}=\sum_{j=1}^{\infty} n^{3} \frac{e^{-2 \kappa r_{1} / n}}{(2 r)^{3}}\left[\left(j-1+c_{x}\right)^{2}\left(\frac{3}{r^{2}}+\frac{6 \kappa}{n r}+\frac{4 \kappa^{2}}{n^{2}}\right)\right. \\
& \left.-\left(1+\frac{2 \kappa r_{1}}{n}\right)\right]\left\{\exp \left[i k L\left(j+c_{x}\right)\right]\right\}, \\
& B_{8}=\sum_{j=1}^{\infty} n^{3} \frac{e^{-2 \kappa r_{1} / n}}{\left(2 r_{1}\right)^{3}}\left[\frac{3 c_{y}^{2}}{r_{1}^{2}}+\frac{4 \kappa^{2} c_{y}^{2}}{n^{2}}+\frac{6 \kappa c_{y}^{2}}{n r_{1}}\right. \\
& \left.-\left(1+\frac{\kappa r_{1}}{n}\right)\right]\left\{\exp \left[i k L\left(j+c_{x}\right)\right]\right\},
\end{aligned}
$$

where $r=\sqrt{\left(j-c_{x}\right)^{2}+c_{y}^{2}}, r_{1}=\sqrt{\left(j-1+c_{x}\right)^{2}+c_{y}^{2}}$, the dimensionless wave number $k$ is in units of $2 \pi / L, i=\sqrt{-1}$, and sub $=8 V_{0} \pi^{2} \cos \left(\pi c_{x}\right)$. 
*joaoclaudio@fisica.ufc.br

†wandemberg@fisica.ufc.br

${ }^{\ddagger}$ gil@fisica.ufc.br

$\S$ francois.peeters@ua.ac.be

${ }^{1}$ H. H. von Grünberg and J. Baumgartl, Phys. Rev. E 75, 051406 (2007).

${ }^{2}$ A. Chowdhury, B. J. Ackerson, and N. A. Clark, Phys. Rev. Lett. 55, 833 (1985).

${ }^{3}$ C. Bechinger, M. Brunner, and P. Leiderer, Phys. Rev. Lett. 86, 930 (2001).

${ }^{4}$ C. J. O. Reichhardt and C. Reichhardt, J. Phys. A 36, 5841 (2003).

${ }^{5}$ C. Reichhardt and C. J. Olson, Phys. Rev. Lett. 88, 248301 (2002).

${ }^{6}$ J. Baumgartl, Ph.D. thesis, University of Stuttgart, 2002.

${ }^{7}$ M. Mikulis, C. J. Olson Reichhardt, C. Reichhardt, R. T. Scalettar, and G. T. Zimanyi, J. Phys. Condens. Matter 16, 7909 (2004).

${ }^{8}$ M. Brunner and C. Bechinger, Phys. Rev. Lett. 88, 248302 (2002).

${ }^{9}$ A. M. Alsayed, M. F. Islam, J. Zhang, P. J. Collings, and A. G. Yodh, Science 309, 1207 (2005).

${ }^{10} \mathrm{~S}$. Herrera-Velarde and R. Castaneda Priego, J. Phys. Condens. Matter 19, 226215 (2007).

${ }^{11}$ S. Herrera-Velarde and R. Castaneda Priego, Phys. Rev. E 77, 041407 (2008).

${ }^{12}$ J. H. Chu and L. I, Phys. Rev. Lett. 72, 4009 (1994).

${ }^{13}$ B. Liu, K. Avinash, and J. Goree, Phys. Rev. Lett. 91, 255003 (2003).

${ }^{14}$ B. Liu and J. Goree, Phys. Rev. E 71, 046410 (2005).

${ }^{15}$ K. Zahn, R. Lenke, and G. Maret, Phys. Rev. Lett. 82, 2721 (1999).

${ }^{16}$ M. Golosovsky, Y. Saado, and D. Davidov, Phys. Rev. E 65, 061405 (2002).

${ }^{17}$ P. Glasson, V. Dotsenko, P. Fozooni, M. J. Lea, W. Bailey, G. Papageorgiou, S. E. Andresen, and A. Kristensen, Phys. Rev. Lett. 87, 176802 (2001).

${ }^{18}$ D. Rees and K. Kono, J. Low Temp. Phys. 158, 301 (2010).

${ }^{19}$ A. Homann, A. Melzer, S. Peters, and A. Piel, Phys. Rev. E 56, 7138 (1997).
${ }^{20}$ T. Misawa, N. Ohno, K. Asano, M. Sawai, S. Takamura, and P. K. Kaw, Phys. Rev. Lett. 86, 1219 (2001).

${ }^{21}$ B. Liu and J. Goree, Phys. Rev. E 71, 046410 (2005).

${ }^{22}$ A. Melzer, Phys. Rev. E 73, 056404 (2006).

${ }^{23}$ T. E. Sheridan and K. D. Wells, Phys. Rev. E 81, 016404 (2010).

${ }^{24}$ M. Koppl, P. Henseler, A. Erbe, P. Nielaba, and P. Leiderer, Phys. Rev. Lett. 97, 208302 (2006).

${ }^{25}$ K. Mangold, J. Birk, P. Leiderer, and C. Bechinger, Phys. Chem. Chem. Phys. 6, 1623 (2004).

${ }^{26}$ G. Piacente, I. V. Schweigert, J. J. Betouras, and F. M. Peeters, Phys. Rev. B 69, 045324 (2004).

${ }^{27}$ G. Piacente, G. Q. Hai, and F. M. Peeters, Phys. Rev. B 81, 024108 (2010).

${ }^{28}$ W. P. Ferreira, J. C. N. Carvalho, P. W. S. Oliveira, G. A. Farias, and F. M. Peeters, Phys. Rev. B 77, 014112 (2008).

${ }^{29}$ W. P. Ferreira, G. A. Farias, and F. M. Peeters, J. Phys. Condens. Matter 22, 11627 (2010).

${ }^{30}$ L. Candido, J. P. Rino, N. Studarta, and F. M. Peeters, J. Phys. Condens. Matter 10, 11627 (1998).

${ }^{31}$ Y. I. Frenkel and T. Kontorova, Zh. Eksp. Teor. Fiz. 8, 1340 (1938).

${ }^{32}$ P. M. Chaikin and T. C. Lubensky, Principles of Condensed Matter Physics, 1st ed. (Cambridge University Press, Cambridge, 2000).

${ }^{33} \mathrm{~S}$. Aubry, in Solitons and Condensed Matter Physics, edited by A. R. Bishop and T. Schneider (Springer, Berlin, 1979).

${ }^{34}$ J. M. Tamga, M. Remoissenet, and J. Pouget, Phys. Rev. Lett. 75, 357 (1995).

${ }^{35}$ P. S. Lomdahl and D. J. Srolovitz, Phys. Rev. Lett. 57, 2702 (1986).

${ }^{36}$ D. J. Srolovitz and P. S. Lomdahl, Physica D 23, 402 (1986).

${ }^{37}$ O. M. Braun and Y. S. Kivshar, The Frenkel-Kontorova Model Concepts, Methods and Applications (Springer, Berlin, 2004).

${ }^{38}$ J. Ou, S. J. Carpenter, and K. D. Dorfman, Biomicrofluidics 4, 013203 (2010).

${ }^{39}$ P. S. Doyle, J. Bibette, A. Bancaud, and J. L. Viovy, Science 295, 2237 (2002).

${ }^{40}$ P. L. Christiansen, A. V. Savin, and A. V. Zolotaryuk, Phys. Rev. B 54, 12892 (1996). 